\title{
Neuropathic Pain and its Relationship With Fibromyalgia, Vitamin D Status and Medication Use in Patients With Ankylosing Spondylitis
}

\author{
Sema Mızrak, M.D. ${ }^{1}$, Mehmet Kırnap, M.D. ${ }^{2}$, İsa Cüce, M.D. ${ }^{2}$ \\ ${ }^{1}$ Şebinkarahisar State Hospital, Department of Physical Medicine and Rehabilitation, Giresun, ${ }^{2}$ Department of Physical Medicine and \\ Rehabilitation, Faculty of Medicine, Erciyes University, Kayseri, Turkey
}

\begin{abstract}
Objective. To determine the frequency of neuropathic pain (NeP) and potentially related new factors including fibromyalgia, vitamin D and medication use in ankylosing spondylitis (AS) patients. Methods. In total, 102 patients with AS were prospectively enrolled in this study and evaluated for pain severity (visual analog scale, VAS), disease activity (the Bath Ankylosing Spondylitis Disease Activity Index, BASDAI), fibromyalgia and current medication use. The presence of NeP was also assessed using the painDETECT questionnaire. Blood samples were taken from all patients to analyze serum 25-hydroxyvitamin $\mathrm{D}$ and inflammatory marker levels. Results. NeP component 32 (21 [20.6\%]; clearly NeP and 11 [10.8\%]; mixed NeP) was present in patients with AS. Compared to those without NeP, they had significantly higher VAS and BASDAI scores $(p=0.022$ and 0.003 , respectively). In addition, there was a highly significant difference of frequency of fibromyalgia between patients with and without $\mathrm{NeP}(50.0 \%$ vs. $5.7 \%, \mathrm{p}<0.001)$. Vitamin $\mathrm{D}$ status and medication use were comparable for patients with and without NeP. Logistic regression analysis revealed that only fibromyalgia was a significant predictor of NeP. Conclusion. This study confirmed that about one-third of AS patients have the NeP component. In addition, NeP was found to be associated with the frequency of fibromyalgia. However, no relation was found between NeP and vitamin D status and medication use in AS. (J Rheum Dis 2021;28:126-132)
\end{abstract}

Key Words. Ankylosing spondylitis, Neuropathic pain, Vitamin D, Fibromyalgia

\section{INTRODUCTION}

Ankylosing spondylitis (AS) is a prevalent form of axial spondyloarthritis (axSpA), with a remarkable link with HLA-B27 [1]. Axial inflammation and new bone formation are two major issues in the pathogenesis of AS. These two ongoing processes are often responsible for chronic back pain and loss of mobility and function in patients with AS. Other common findings related to pain are asymmetric oligoarthritis and enthesitis [2].

Although the pain in AS has an inflammatory nature, recent studies have suggested that neuropathic pain ( $\mathrm{NeP})$ component exists in $25 \% \sim 35.4 \%$ of patients [3-5]. A structural magnetic resonance study also revealed the occurrence of additional structural brain abnormalities, which are compatible with neural correlations of $\mathrm{NeP}$, in patients with AS, thereby supporting the existence of a central pain process [6]. NeP is thought to be caused by the immune response due to chronic stimulation of the joints and entheses, leading to neural plasticity in the peripheral and central nervous systems [7]. Current treatments of AS, including the use of biological agents, primarily focus on suppressing inflammation; however, they do not always provide satisfactory pain relief in all patients. One of the reasons for this is that comorbidities associated with accompanying pain in these patients fur-

\footnotetext{
Received : December 28, 2020, Revised : March 13, 2021, Accepted : March 25, 2021

Corresponding to : İsa Cüce (iD http://orcid.org/0000-0002-1290-9540

Department of Physical Medicine and Rehabilitation, Faculty of Medicine, Erciyes University, Köşk Mah. Dede Efendi Sok. P.K. 38030 Melikgazi, Kayseri, Turkey. E-mail : dr.icuce@hotmail.com
}

Copyright (C) 2021 by The Korean College of Rheumatology.

This is an Open Access article, which permits unrestricted non-commerical use, distribution, and reproduction in any medium, provided the original work is properly cited. 
ther complicate neuro-immune interactions and affect the character of the current pain $[8,9]$.

Previous studies have reported that NeP in AS is associated with demographic, disease-related (i.e., disease activity or enthesitis) and emotional factors [3-5]. However, other factors affecting pain in AS patients, which are common and potentially associated with $\mathrm{NeP}$, have not been adequately investigated. First, vitamin D deficiency is frequent among patients with AS and potentially related to pain, whereas the impact of vitamin D status on AS-related $\mathrm{NeP}$ is unknown [10]. The correlation of the presence and severity of neuropathy with a poor vitamin $\mathrm{D}$ status in painful neuropathic diseases has been demonstrated in recent studies [11,12]. Second, in a recent systemic review of axSpA, concomitant fibromyalgia has reported in $13.8 \%$ of patients with AS [13]. Studies indicate that patients with axSpA with concomitant fibromyalgia have higher disease burden with higher pain severity and lower quality of life than those without $[14,15]$. Although pain in fibromyalgia is not included in the NeP classification, recent studies containing primary data on pathophysiology have highlighted the neuropathic component of pain in fibromyalgia, and even the new category as "centralized pain" is proposed [16-18]. Moreover, the effect of concomitant fibromyalgia on $\mathrm{NeP}$ in patients with axSpA or other inflammatory arthritis is not clear. Therefore, the purpose of this study is to investigate the frequency of $\mathrm{NeP}$ in AS and its relationship with some new potential primary data added.

\section{MATERIALS AND METHODS}

This cross-sectional study was conducted between November 2018 and October 2019 at our department's outpatient clinic. The study protocol received ethics approval from the local ethics committee (reference number: 2018/542). All the patients received detailed information about the study protocol and provided signed consent. According to the modified New York criteria, patients 18 years and older diagnosed with AS were consecutively enrolled during their medical appointments. The exclusion criteria were as follows: (i) the presence of accompanying systemic or neurological disorders that can cause NeP, e.g., diabetes mellitus, chronic renal impairment, or polyneuropathy; (ii) the presence of metabolic bone diseases; (iii) the presence of psychiatric disorders; (iv) the use of medications affecting $\mathrm{NeP}$ or vitamin $\mathrm{D}$ levels, e.g., antidepressants, antiepileptics, or calcium and vitamin D or B12 supplements; (v) chronic alcohol or drug misuse; (vi) a previous diagnosis of vitamin B12 deficiency; and (vii) refusal to participate in the study.

A standardized form was used to gather demographic and disease related data, including age, gender, body length and body weight, educational status, presence of systemic diseases, disease duration, and current medication and duration of use. The patients were questioned for the presence of concomitant fibromyalgia, and noted. The American College of Rheumatology (ACR) 2010 classification criteria were used to diagnose fibromyalgia.

The overall pain intensity of the patients within the last week was evaluated using a horizontal visual analog scale (VAS) scoring system. The disease activity was tested using the Bath Ankylosing Spondylitis Disease Activity Index (BASDAI), which is a simple and valid instrument that includes five main disease parameters, namely fatigue/tiredness, back/hip pain, peripheral joint pain/swelling, localized tenderness, and morning stiffness [19]. The total scores range from 0 to 10 , with higher scores showing increased disease activity.

The neuropathic component of the pain was assessed with the painDETECT questionnaire (PD-Q). In adults, the PD-Q questionnaire used to detect NeP components in chronic painful conditions, developed by Freynhagen et al. [20]. The survey contains seven items relevant to the nature and intensity of specific NeP symptoms. The total score ranges from -1 to 38 , with $<13,13 \sim 18$, and $\geq 19$ points representing "unlikely," "mixed" and "very likely" $\mathrm{NeP}$ component, respectively. The PD-Q can be easily administered and validated in Turkish [21].

A total of $5 \mathrm{~mL}$ of fasting peripheral blood was collected early in the morning. Serum 25-hydroxyvitamin D $(25(\mathrm{OH}) \mathrm{D})$ levels were quantitatively determined using liquid chromatography/tandem mass spectrometry method. The vitamin D status was considered sufficient, insufficient, and deficient for patients with serum $25(\mathrm{OH}) \mathrm{D}$ levels $\geq 30 \mathrm{ng} / \mathrm{mL}$, between 21 and $29 \mathrm{ng} / \mathrm{mL}$, and $\leq 20 \mathrm{ng} / \mathrm{mL}$, respectively [22]. Additional laboratory tests were carried out, including erythrocyte sedimentation rate (ESR) and C-reactive protein (CRP).

\section{Statistical analysis}

Data analyses were performed using SPSS 26.0 statistical software (IBM Co., Armonk, NY, USA). The basic characteristics of the patients were evaluated using descriptive statistics. The Kolmogorov-Smirnov test and graphical methods were used to check the normal dis- 
tribution of quantitative data. Based on the total PD-Q score, the patients divided into NeP-negative (NePN, $<13$ points) and NeP-positive groups (NePP, $\geq 13$ points). For comparison of continuous variables between NePP and NePN groups, Student's t-test or MannWhitney U-test (if t-test assumptions were not met) was used. Univariate and multivariate analyses were performed to define NEP-related independent predictors for AS. The significance level was accepted at $\mathrm{p}<0.05$.

\section{RESULTS}

During the study period, 125 patients diagnosed with AS were enrolled; and eventually, 102 patients met eligibility criteria. Of 102 patients, 54 (52.4\%) were males and $48(46.6 \%)$ were females. The mean age of patients was $40.5 \pm 10.9$ years. The demographic and clinical data of the AS patients are shown in Table 1.

Based on their PD-Q score, $31.4 \%$ of patients had the
$\mathrm{NeP}$ component (21 [20.6\%]; clearly NeP and 11 [10.8\%]; mixed NeP); $68.6 \%$ had no NeP component. The clinical data for patients with and without NeP are presented in Table 1. In comparing the NePP and NEPN groups, there were no significant differences in age, gender and disease duration. However, VAS and BASDAI scores were significantly higher in the NePP group than in the NePN group ( $p=0.022$ and 0.003 , respectively). The serum $25(\mathrm{OH}) \mathrm{D}$ levels were low in both the groups, and the between-group difference did not reach statistical significance $(p=0.329)$. The two groups were also comparable in terms of vitamin $D$ status $(p=0.973)$. The frequency of fibromyalgia was $50.0 \%$ and $5.7 \%$, respectively, in NePP and NePN groups, and this difference was highly significant $(\mathrm{p}<0.001)$.

Correlation analysis showed that PD-Q scores was positively correlated with VAS and BASDAI $(\mathrm{p}<0.001$, $\mathrm{r}=0.658$ and $\mathrm{p}<0.001, \mathrm{r}=0.633$, respectively), and negatively correlated with duration of tumor necrosis factor

Table 1. Demographic and clinical variables in patients with AS $(n=102)$ based on PD-Q score

\begin{tabular}{|c|c|c|c|c|}
\hline Variable & NePN group $(n=70)$ & NePP group $(n=32)$ & Total $(n=102)$ & p-value \\
\hline Age (yr) & $40.33 \pm 11.20$ & $40.81 \pm 10.34$ & $40.5 \pm 10.9$ & 0.836 \\
\hline \multicolumn{5}{|l|}{ Sex } \\
\hline Male & $35(50.0)$ & $19(59.4)$ & $54(52.9)$ & \multirow[t]{2}{*}{0.379} \\
\hline Female & $35(50.0)$ & $13(40.6)$ & $48(47.1)$ & \\
\hline \multicolumn{5}{|l|}{ BMI $\left(\mathrm{kg} / \mathrm{m}^{2}\right)$} \\
\hline$<25$ & $29(41.4)$ & $12(37.5)$ & $41(40.2)$ & \multirow[t]{2}{*}{0.707} \\
\hline$\geq 25$ & $41(58.6)$ & $20(62.5)$ & $61(59.8)$ & \\
\hline Disease duration (yr) & $9.55 \pm 8.87$ & $7.94 \pm 6.39$ & $9.04 \pm 8.18$ & 0.358 \\
\hline \multicolumn{5}{|l|}{ AS medication } \\
\hline NSAID & $17(24.3)$ & 7 (21.9) & $24(23.5)$ & \multirow[t]{4}{*}{0.326} \\
\hline DMARD (alone or combination) & $4(5.7)$ & $5(15.6)$ & $9(8.8)$ & \\
\hline TNF inhibitor & $43(61.4)$ & $19(59.4)$ & $62(60.8)$ & \\
\hline None & $6(8.6)$ & $1(3.1)$ & $7(6.9)$ & \\
\hline Duration of TNF inhibitors, months & $45.1 \pm 27.1$ & $32.1 \pm 28.2$ & $41.1 \pm 27.9$ & 0.089 \\
\hline VAS pain & $3.84 \pm 2.42$ & $5.03 \pm 2.32$ & $4.22 \pm 2.44$ & 0.022 \\
\hline BASDAI & $3.19 \pm 2.28$ & $4.66 \pm 2.29$ & $3.64 \pm 2.37$ & 0.003 \\
\hline $\mathrm{ESR}(\mathrm{mm} / \mathrm{h})$ & $11.56 \pm 9.68$ & $12.09 \pm 10.43$ & $11.7 \pm 9.9$ & 0.800 \\
\hline $\mathrm{CRP}(\mathrm{mg} / \mathrm{dL})$ & $9.30 \pm 13.06$ & $7.06 \pm 7.71$ & $8.6 \pm 11.7$ & 0.371 \\
\hline $25(\mathrm{OH}) \mathrm{D}(\mathrm{ng} / \mathrm{mL})$ & $19.36 \pm 7.06$ & $17.78 \pm 8.37$ & $18.9 \pm 7.5$ & 0.329 \\
\hline \multicolumn{5}{|l|}{ Vitamin D status } \\
\hline$\leq 20 \mathrm{ng} / \mathrm{mL}$ & $40(57.1)$ & $19(59.4)$ & $59(57.8)$ & \multirow[t]{3}{*}{0.973} \\
\hline $21 \sim 29 \mathrm{ng} / \mathrm{mL}$ & $25(35.7)$ & $11(34.4)$ & $36(35.3)$ & \\
\hline$\geq 30 \mathrm{ng} / \mathrm{mL}$ & $5(7.1)$ & $2(6.3)$ & $7(6.9)$ & \\
\hline Fibromyalgia & $4(5.7)$ & $16(50.0)$ & $20(19.6)$ & $<0.001$ \\
\hline
\end{tabular}

Data were expressed as mean \pm standard deviation or frequency (\%). AS: ankylosing spondylitis, PD-Q: painDETECT questionnaire, NePN: neuropathic pain-negative, NePP: neuropathic pain-positive, BMI: body mass index, NSAID: nonsteroidal antiinflammatory drug, DMARD: disease-modifying antirheumatic drug, VAS: visual analog scale, BASDAI: Bath Ankylosing Spondylitis Disease Activity Index, ESR: erythrocyte sedimentation rate, CRP: C-reactive protein, 25(OH)D: 25-hydroxyvitamin D. 
Table 2. Factors associated with NeP by univariate and multivariate logistic regression analysis

\begin{tabular}{|c|c|c|c|c|c|c|}
\hline \multirow{2}{*}{ Variable } & \multicolumn{3}{|c|}{ Univariate analysis } & \multicolumn{3}{|c|}{ Multivariate analysis } \\
\hline & OR & $95 \% \mathrm{Cl}$ & p-value & OR & $95 \% \mathrm{Cl}$ & p-value \\
\hline Age & 1.004 & $0.966 \sim 1.044$ & 0.834 & & & \\
\hline Sex & 1.462 & $0.627 \sim 3.409$ & 0.380 & & & \\
\hline $\mathrm{BMI}$ & 1.179 & $0.499 \sim 2.784$ & 0.707 & & & \\
\hline CRP & 0.981 & $0.940 \sim 1.024$ & 0.375 & & & \\
\hline Disease duration & 0.974 & $0.922 \sim 1.030$ & 0.356 & & & \\
\hline AS medication & 1.114 & $0.736 \sim 1.686$ & 0.609 & & & \\
\hline Fibromyalgia & 16.500 & $4.851 \sim 56.126$ & $<0.001$ & 16.500 & $4.851 \sim 56.126$ & $<0.001$ \\
\hline $25(\mathrm{OH}) \mathrm{D}$ & 0.971 & $0.917 \sim 1.029$ & 0.326 & & & \\
\hline BASDAI & 1.315 & $1.087 \sim 1.591$ & 0.005 & & & \\
\hline VAS & 1.233 & $1.027 \sim 1.481$ & 0.025 & & & \\
\hline
\end{tabular}

NeP: neuropathic pain, OR: odds ratio, $\mathrm{Cl}$ : confidence interval, BMI: body mass index, CRP: C-reactive protein, 25(OH)D: 25-hydroxyvitamin D, BASDAI: Bath Ankylosing Spondylitis Disease Activity Index, VAS: visual analog scale.

(TNF) inhibitors $(\mathrm{p}=0.039, \mathrm{r}=-0.262)$. However, PD-Q scores were not significantly correlated with age $(p=0.767)$, disease duration $(p=0.085)$ and $25(\mathrm{OH}) \mathrm{D}$ levels $(p=0.489)$. In logistic regression, only fibromyalgia was found to be statistically significant as a predictor of $\mathrm{NeP}$ (odds ratio [OR], 16.500; 95\% confidence interval, 4.851 56.126) (Table 2).

\section{DISCUSSION}

Our study provides new insights into the growing literature suggesting that the $\mathrm{NeP}$ component is present in patients with axSpA. Of the 102 patients with AS included in the study, 21 (20.6\%) clearly (very likely) had the NeP component (PD-Q score $\geq 19$ points). Furthermore, the possible NeP component (PD-Q scores between 13 and 18 points), i.e., mixed pain, was present in $10.8 \%$ of the patients. Similar results were obtained in previous studies investigating the prevalence of $\mathrm{NeP}$ in patients with AS using the PD-Q [3-5]. These studies revealed the presence of the $\mathrm{NeP}$ component in $25 \%, 32.8 \%$, and $35.2 \%$ of patients with AS, respectively. In addition, using the PD-Q, Wu et al. [6], detected the NeP component in 11 out of 17 AS patients with active disease and back pain. A recently published meta-analysis showed that AS patients with $\mathrm{NeP}$ had higher pain intensity, higher disease activity, and poorer quality of life than those without NeP. In addition, NeP was higher in females than in males. Moreover, HLA-B27 was present in significantly more patients with NeP, compared to those without NeP. In contrast, age, body mass index, symptom duration, and levels of systemic markers of inflammation (i.e., ESR and CRP level) did not differ between patients with and without $\mathrm{NeP}$ [23]. The findings in our study were consistent, except for the gender ratio.

Fibromyalgia was considerably more frequent in patients with $\mathrm{NeP}$, and it was revealed to be an independent predictor of $\mathrm{NeP}$ by logistic regression analysis. Considering the current research on NeP in AS patients, it seems confusing whether the presence of fibromyalgia in these patients can be excluded. Choi et al. [5] reported that concomitant fibromyalgia in AS patients with $\mathrm{NeP}$ was substantially higher, but it was not an independent predictor of NeP. Gok et al. [4] found similar frequency of fibromyalgia in patients with the NeP component compared to patients without it. On the other hand, in the study conducted by Geler-Külcü et al. [3], patients with AS with concomitant fibromyalgia, despite having similar methodology, were excluded. In our study, $19.6 \%$ of patients had fibromyalgia according to the ACR 2010 fibromyalgia criteria, and more than half of them had a NeP component. The total number of female patients included in the study was relatively high, but 12 of the 20 patients with concomitant fibromyalgia were females. Depending on the criteria used, the frequency of concomitant fibromyalgia was found to be between $4 \%$ and $25 \%$ in patients with AS [24]. However, in another study, interestingly, the frequency of fibromyalgia was found to be $19 \%$ $29 \%$ by applying the ACR 1990 and 2010 FM criteria to the same AS cohort [25]. Using the PD-Q, a recent study revealed that $52.5 \%$ of the patients with fibromyalgia had a NeP component [18]. On the other hand, the PD-Q has been found to have low sensitivity and specificity in distinguishing $\mathrm{NeP}$ in patients with fibromyalgia [26]. 
Understanding the complex relationship between fibromyalgia and $\mathrm{NeP}$ is challenging because of their clinical and neurobiological similarities [27]. A study using patient reported questionnaires in patients with diabetic polyneuropathy and fibromyalgia showed that both groups of patients experienced very similar sensory symptoms and that sensory profiles were overlapping in $20 \% \sim 35 \%$ of patients [28]. The presence of NeP in inflammatory arthritis is quite new to diabetic polyneuropathy, and self-assessment questionnaires have generally been used in current research; however, our knowledge of their uses and validity is very limited. Therefore, in cross-sectional studies, it may be not possible to determine whether the $\mathrm{NeP}$ component in patients with AS is related to the disease itself or to fibromyalgia and whether fibromyalgia or AS contributes to the patients' current $\mathrm{NeP}$ using self-administered questionnaires, such as the PD-Q.

We found no substantial difference in the vitamin $\mathrm{D}$ levels or status (sufficiency, insufficiency, and deficiency) between AS patients with and without NeP. Most research has primarily addressed the relationships between diabetic peripheral neuropathy and vitamin $\mathrm{D}$. The results of a recent meta-analysis showed that there is a close relationship between vitamin $\mathrm{D}$ and diabetic peripheral neuropathy, and vitamin $\mathrm{D}$ deficiency is one of the risk factors for neuropathy [29]. Moreover, some previous studies have reported that vitamin D replacement treatment has beneficial effects on neuropathic signs/symptoms in patients with diabetic NeP and vitamin D deficiency $[30,31]$. However, in a recently published study, possible NeP was detected in $26.8 \%$ of 236 diabetic patients using the PD-Q; no significant relationship was observed when comparing the vitamin $\mathrm{D}$ status of patients with and without NeP. The authors interpreted incompatibility with previous studies as a result of the limited sample size and the use of a different neuropathy assessment tool (i.e., the PD-Q) [32]. Yesil et al. [33] detected $\mathrm{NeP}$ in $33.3 \%$ of 93 patients with rheumatoid arthritis (RA) using the Leeds Assessment of Neuropathic Symptoms and Signs (LANSS) pain scale. In their study, the mean serum 25(OH)D level was lower than $20 \mathrm{ng} / \mathrm{mL}$ in patients with $\mathrm{NeP}$ and higher than $20 \mathrm{ng} / \mathrm{mL}$ in patients without $\mathrm{NeP}$, and there was a significant difference between the two groups. Furthermore, the prevalence of $\mathrm{NeP}$ was about six times greater in patients with vitamin $D$ deficiency than in those with sufficient levels of vitamin D. In contrast to RA, the lack of a reasonable association between $\mathrm{NeP}$ and vitamin $\mathrm{D}$ deficiency in patients with AS could be attributed to the relatively small number of patients with $\mathrm{NeP}$ as well as the methodological differences in our study.

In our study, we found no significant difference in medication use between AS patients with and without NeP. The frequency of those using NSAIDs or TNF inhibitors was similar in both groups. There was only a weak negative correlation with the PD-Q scores and the duration of TNF inhibitors. Approximately two-thirds of all patients in our study had been using TNF inhibitors for a long period (at least 6 months). Wu et al. [34] demonstrated that TNF inhibitors could reduce NeP severity in a small proportion of AS patients with NeP. Similarly, in our study, the severity of pain and PD-Q scores of the patients using TNF inhibitors were lower in the NeP group compared to those who did not (data not shown). In a recently published study, almost all 58 AS patients were taking NSAIDs, while 22 patients were taking TNF inhibitors. In this study, NeP was detected in $50 \%$ of the patients [35]. Thus, although anti-inflammatory agents appear to alleviate NeP severity in patients with AS, this findings confirm that they are not sufficient for $\mathrm{NeP}$ treatment.

Several limitations of the present study need to be noted. We used a self-assessment screening (and not diagnostic) questionnaire to evaluate the $\mathrm{NeP}$ component of patients. Therefore, it cannot replace the clinical history and examination, which also assess the functions of the somatosensory system. In addition, the fact that only 32 patients had NeP component may have weakened the strength of logistic regression to evaluate variables. As far as we know, our study is the first to explore the relationship between $\mathrm{NeP}$ and vitamin $\mathrm{D}$ status in patients with AS. However, our results should be viewed with caution because of the cross-sectional study design. Longitudinal studies are required in patients with AS to validate potential causal relationships between $\mathrm{NeP}$ and vitamin D status or medication use.

\section{CONCLUSION}

This study confirmed that This study confirmed that about one-third of AS patients have the NeP component. In addition, $\mathrm{NeP}$ was found to be associated with the frequency of fibromyalgia. However, no relation was found between $\mathrm{NeP}$ and vitamin $\mathrm{D}$ status and medication use in AS. Furthermore, the pain severity and disease activity were higher in patients with $\mathrm{NeP}$ than in those without 
$\mathrm{NeP}$. Further research is necessary to confirm our findings and related mechanisms.

\section{ACKNOWLEDGMENTS}

This research was supported by Erciyes University Research Foundation (Project Number TTU-2019-9511).

\section{CONFLICT OF INTEREST}

No potential conflict of interest relevant to this article was reported.

\section{AUTHOR CONTRIBUTIONS}

Conceptualization: S.M. and M.K. Data acquisition: S.M., M.K., and I.C. Formal analysis: S.M and I.C. Funding: Erciyes University. Supervision: M.K. Writingoriginal draft: S.M. and İ.C. Writing-review \& editing: İ.C. and M.K.

\section{REFERENCES}

1. Carli L, Calabresi E, Governato G, Braun J. One year in review 2018: axial spondyloarthritis. Clin Exp Rheumatol 2019;37:889-98.

2. Kiltz U, Baraliakos X, Regel A, Bühring B, Braun J. Causes of pain in patients with axial spondyloarthritis. Clin Exp Rheumatol 2017;35 Suppl 107:102-7.

3. Geler-Külcü D, Batıbay S, Öztürk G, Mesci N. The association of neuropathic pain and disease activity, functional level, and quality of life in patients with ankylosing spondylitis: a cross-sectional study. Turk J Med Sci 2018;48:257-65.

4. Gok K, Cengiz G, Erol K, Ozgocmen S. Neuropathic pain component in axial spondyloarthritis and the influence on disease burden. J Clin Rheumatol 2018;24:324-7.

5. Choi JH, Lee SH, Kim HR, Lee KA. Association of neuropathic-like pain characteristics with clinical and radiographic features in patients with ankylosing spondylitis. Clin Rheumatol 2018;37:3077-86.

6. Wu Q, Inman RD, Davis KD. Neuropathic pain in ankylosing spondylitis: a psychophysics and brain imaging study. Arthritis Rheum 2013;65:1494-503.

7. Lampa J. Pain without inflammation in rheumatic diseases. Best Pract Res Clin Rheumatol 2019;33:101439.

8. Bidad K, Gracey E, Hemington KS, Mapplebeck JCS, Davis $\mathrm{KD}$, Inman RD. Pain in ankylosing spondylitis: a neuro-immune collaboration. Nat Rev Rheumatol 2017;13:410-20.

9. Pathan EMI, Inman RD. Pain in spondyloarthritis: a neuro-immune interaction. Best Pract Res Clin Rheumatol 2017;31:830-45.

10. Zhao S, Duffield SJ, Moots RJ, Goodson NJ. Systematic review of association between vitamin $\mathrm{D}$ levels and susceptibility and disease activity of ankylosing spondylitis. Rheumatology
(Oxford) 2014;53:1595-603.

11. Celikbilek A, Gocmen AY, Tanik N, Borekci E, Adam M, Celikbilek M, et al. Decreased serum vitamin D levels are associated with diabetic peripheral neuropathy in a rural area of Turkey. Acta Neurol Belg 2015;115:47-52.

12. Chao CT, Chiang CK, Huang JW, Hung KY. Vitamin D is closely linked to the clinical courses of herpes zoster: from pathogenesis to complications. Med Hypotheses 2015;85: $452-7$.

13. Jones GT, Mallawaarachchi B, Shim J, Lock J, Macfarlane GJ. The prevalence of fibromyalgia in axial spondyloarthritis. Rheumatol Int 2020;40:1581-91.

14. Sayın S, Yurdakul FG, Sivas F, Bodur H. Is fibromyalgia frequency increasing in axial spondyloarthritis? Association with fibromyalgia and biological therapies. Rheumatol Int 2020;40:1835-41.

15. Duffield SJ, Miller N, Zhao S, Goodson NJ. Concomitant fibromyalgia complicating chronic inflammatory arthritis: a systematic review and meta-analysis. Rheumatology (Oxford) 2018;57:1453-60.

16. Cheng CW, Wong CS, Hui GK, Chung EK, Wong SH. Fibromyalgia: is it a neuropathic pain? Pain Manag 2018;8: 377-88.

17. Ueda H. Systems pathology of neuropathic pain and fibromyalgia. Biol Pharm Bull 2019;42:1773-82.

18. Kösehasanoğullari M, Erdinç Gündüz N, Akalin E. Is fibromyalgia syndrome a neuropathic pain syndrome? Arch Rheumatol 2018;34:196-203.

19. Zochling J. Measures of symptoms and disease status in ankylosing spondylitis: Ankylosing Spondylitis Disease Activity Score (ASDAS), Ankylosing Spondylitis Quality of Life Scale (ASQoL), Bath Ankylosing Spondylitis Disease Activity Index (BASDAI), Bath Ankylosing Spondylitis Functional Index (BASFI), Bath Ankylosing Spondylitis Global Score (BAS-G), Bath Ankylosing Spondylitis Metrology Index (BASMI), Dougados Functional Index (DFI), and Health Assessment Questionnaire for the Spondylarthropathies (HAQ-S). Arthritis Care Res (Hoboken) 2011;63 Suppl 11:S47-58.

20. Freynhagen R, Baron R, Gockel U, Tölle TR. painDETECT: a new screening questionnaire to identify neuropathic components in patients with back pain. Curr Med Res Opin 2006;22:1911-20.

21. Alkan H, Ardic F, Erdogan C, Sahin F, Sarsan A, Findikoglu G. Turkish version of the painDETECT questionnaire in the assessment of neuropathic pain: a validity and reliability study. Pain Med 2013;14:1933-43.

22. Wacker M, Holick MF. Vitamin D - effects on skeletal and extraskeletal health and the need for supplementation. Nutrients 2013;5:111-48.

23. Kim TW, Son SM, Lee JS. Neuropathic pain in ankylosing spondylitis: a meta-analysis. Z Rheumatol 2020;79:95-102.

24. Zhao SS, Duffield SJ, Goodson NJ. The prevalence and impact of comorbid fibromyalgia in inflammatory arthritis. Best Pract Res Clin Rheumatol 2019;33:101423.

25. Baraliakos X, Regel A, Kiltz U, Menne HJ, Dybowski F, Igelmann $\mathrm{M}$, et al. Patients with fibromyalgia rarely fulfil classification criteria for axial spondyloarthritis. Rheumatology (Oxford) 2018;57:1541-7.

26. Gauffin J, Hankama T, Kautiainen H, Hannonen P, Haanpää M. Neuropathic pain and use of PainDETECT in patients 
with fibromyalgia: a cohort study. BMC Neurol 2013;13:21.

27. Maletic V, Raison CL. Neurobiology of depression, fibromyalgia and neuropathic pain. Front Biosci (Landmark Ed) 2009;14:5291-338.

28. Koroschetz J, Rehm SE, Gockel U, Brosz M, Freynhagen R, Tölle TR, et al. Fibromyalgia and neuropathic pain--differences and similarities. A comparison of 3057 patients with diabetic painful neuropathy and fibromyalgia. BMC Neurol 2011;11:55.

29. Zhang B, Zhao W, Tu J, Wang X, Hao Y, Wang H, et al. The relationship between serum 25-hydroxyvitamin D concentration and type 2 diabetic peripheral neuropathy: a systematic review and a meta-analysis. Medicine (Baltimore) 2019;98:e18118.

30. Sari A, Akdoğan Altun Z, Arifoglu Karaman C, Bilir Kaya B, Durmus B. Does vitamin $D$ affect diabetic neuropathic pain and balance? J Pain Res 2020;13:171-9.

31. Lee $P$, Chen R. Vitamin $D$ as an analgesic for patients with type 2 diabetes and neuropathic pain. Arch Intern Med 2008;168:771-2.

32. Alkhatatbeh M, Abdul-Razzak KK. Neuropathic pain is not associated with serum vitamin $\mathrm{D}$ but is associated with female gender in patients with type 2 diabetes mellitus. BMJ Open Diabetes Res Care 2019;7:e000690.

33. Yesil H, Sungur U, Akdeniz S, Gurer G, Yalcın B, Dundar U. Association between serum vitamin $\mathrm{D}$ levels and neuropathic pain in rheumatoid arthritis patients: a cross-sectional study. Int J Rheum Dis 2018;21:431-9.

34. Wu Q, Inman RD, Davis KD. Tumor necrosis factor inhibitor therapy in ankylosing spondylitis: differential effects on pain and fatigue and brain correlates. Pain 2015;156: 297-304.

35. Borman P, Kaygisiz F, Yaman A. Neuropathic component of low back pain in patients with ankylosing spondylitis. Mod Rheumatol 2021;31:462-7. 